\title{
Do Infants Die of Sudden Infant Death Syndrome (SIDS) With Long QT Syndrome (LQTS) or From LQTS?
}

\author{
David T. Mage
}

Received: 27 June 2012/ Accepted: 13 July 2012/Published online: 7 August 2012

(C) Springer Science+Business Media, LLC 2012

Letter to the Editor,

Tester and Ackerman [4] discuss the use of a "molecular autopsy" for victims of sudden infant death syndrome (SIDS) to determine whether their deaths could have been caused by the long QT syndrome (LQTS). They estimate in their Fig. 1 that $10 \%$ of all such SIDS deaths actually are caused by cardiac channelopathies resulting in LQTS. However, this estimate may be too high for the following two reasons:

(1) The presence of a genetic susceptibility to a sudden cardiac death (SCD) by LQTS does not prevent an infant from dying of nonchannelopathic causes such as positional asphyxiation while sleeping prone with the head partially covered and so forth. Thus, if a genetic marker is present, there is no way to discern whether the infant died of SIDS with LQTS susceptibility or from SCD by LQTS.

(2) Universally, SIDS has approximately a $50 \%$ male excess corresponding to a male fraction of about 0.60 for all races combined [2]. However, Stramba-Badiale et al. [3] have shown "that gender-related differences in QTc observed in the adult population are not present at birth". This gender independence in the first year of life has been confirmed recently by Costa et al. [1] who showed in their Fig. 2 that during the first year of life, males and females have identical rates of death by LQTS.

It is therefore suggested that the $10 \%$ of LQTS among SIDS cases may be an overestimate.

\section{References}

1. Costa $\mathbf{J}$ et al (2012) Combined assessment of sex- and mutationspecific information for risk stratification in type 1 long QT syndrome. Heart Rhythm 9:892-898

2. Mage DT, Donner EM (2004) The fifty percent male excess of infant respiratory mortality. Acta Paediatr 93:1210-1215

3. Stramba-Badiale M, Spagnolo D, Bosi G, Schwartz PJ (1995) Are gender differences in QTc present at birth? MISNES Investigators. Multicenter Italian study on neonatal electrocardiography and sudden infant death syndrome. Am J Cardiol 75:1277-1278

4. Tester DJ, Ackerman MJ (2012) The molecular autopsy: should the evaluation continue after the funeral? Pediatr Cardiol 33:461-470

D. T. Mage has retired from World Health Organization.

D. T. Mage $(\bowtie)$

World Health Organization, Newark, DE, USA

e-mail: magedonner@aol.com 